\title{
Plasticity of Neuroblastoma Tumor Cells to Differentiate along a Fetal Adrenal Ganglionic Lineage Predicts for Improved Patient Survival
}

Mark J. Cooper, * Seth M. Steinberg, ${ }^{*}$ Jane Chatten, ${ }^{3}$ Audrey E. Evans, I and Mark A. Israel *

*Pediatric Branch and ${ }^{\ddagger}$ Biostatistics and Data Management Section, National Cancer Institute, National Institutes of Health, Bethesda, Maryland 20892; and Departments of ${ }^{\S}$ Pathology and 'Oncology, Children's Hospital of Philadelphia, Philadelphia, Pennsylvania 19104

\begin{abstract}
We have recently presented a model of human adrenal medullary histogenesis that incorporates all neural crest-derived lineages (chromaffin, sustentacular, and ganglionic) known to compose this tissue. To determine if neuroblastomas correspond to the arrested maturation of embryonal adrenal medullary cells, we evaluated the expression of adrenal medullary developmental markers in 81 neuroblastoma tumors. We found that patterns of chromaffin-related gene expression in these tumors correlated exactly with the patterns observed during maturation of adrenal medullary cells $\left(P_{2}<10^{-5}\right)$. In a multivariate Cox proportional hazards analysis of developmental marker expression and other well-recognized prognostic variables, evidence of maturation along a fetal ganglionic lineage, as monitored by HNK-1 immunoreactivity (relative risk of 6.42, $P_{2}=0.0001$ ), and age at diagnosis (relative risk of 5.05, $P_{2}=0.0042$ ) were independent and significant prognostic indicators of patient survival. These studies demonstrate that neuroblastomas correspond to embryonal adrenal medullary cells arrested at recognizable stages during development, and that evidence of maturation along a fetal ganglionic lineage appears to have major importance in predicting patient survival. ( $J$. Clin. Invest. 1992. 90:2402-2408.) Key words: neuroblastoma - differentiation • neural crest • HNK-1 • prognostic marker
\end{abstract}

\section{Introduction}

Neuroblastoma, a pediatric tumor arising in tissues derived from the embryonal neural crest, most frequently occurs at known sites of the sympathetic peripheral nervous system (1). This tumor exhibits remarkable heterogeneity in its histopathologic appearance, biochemical properties, and response to chemotherapy (2-8). Although a number of prognostic indicators of patient survival have been described for this tumor, including age of the patient at diagnosis, stage, amplification of the $\mathrm{N}-m y c$ oncogene, the Shimada histopathologic classification,

This study was presented in part at the 26th Meeting of the American Society of Clinical Oncology, Washington, DC, 20-22 May 1990, and was published in abstract form (1990. Proc. Am. Soc. Clin. Oncol. 9:293).

Address correspondence and reprint requests to Mark J. Cooper, M.D., Division of Hematology-Oncology, Ireland Cancer Center, University Hospitals of Cleveland, Case Western Reserve University School of Medicine, 2074 Abington Road, Cleveland, OH 44106. Dr. Israel's current address is Brain Tumor Research Center, HSW 783, University of California at San Francisco, San Francisco, CA 94143. 1992.

Received for publication 24 March 1992 and in revised form 2 July

The Journal of Clinical Investigation, Inc.

Volume 90, December 1992, 2402-2408 expression of neuropeptide $Y$, and serum ferritin levels (6, 9-13), the biological basis for the significance of these prognostic factors remains poorly understood.

Clinical, histopathologic, and laboratory findings suggest that altered regulation of neural crest cell differentiation can play a role in the pathogenesis of neuroblastoma (14-18). For example, the remarkably high incidence of neuroblastoma in situ reported in autopsy series of infants dying from causes unrelated to cancer suggests that many of these in situ tumors spontaneously remit, perhaps by differentiating to mature adrenal medullary tissues $(14,15)$. Similarly, stage IVS neuroblastoma tumors, a variant of stage IV disease presenting itself in patients $<1 \mathrm{yr}$ of age who have small primary tumors and remote disease confined to liver, skin, and/or bone marrow, often undergo spontaneous tumor regression, occasionally resulting in residual, benign ganglioneuromas (19-21).

We therefore investigated the possibility that neuroblastoma tumor cells may correspond to the arrested differentiation of adrenal medullary progenitor cells found during histogenesis of this tissue. In previous studies, we identified and characterized a series of markers defining discrete stages during the maturation of adrenal medullary chromaffin cells and distinguishing cells of this lineage from other neural crest-derived adrenal precursors that differentiate along either the sustentacular or ganglionic lineage $(17,22,23)$. We found that neuroblastoma cell lines express these chromaffin markers in patterns found during development, suggesting that these tumor cells correspond to adrenal medullary chromaffin progenitor cells that are arrested in differentiation (17). In this study, we examined the expression of these lineage-related markers in neuroblastoma tumor specimens and evaluated the clinical implications of such a developmental analysis.

\section{Methods}

Tumor specimens. 81 formalin-fixed, paraffin-embedded neuroblastoma tumor specimens obtained from Children's Hospital of Philadelphia were used in this study. These samples were $85 \%(81 / 95)$ of accrued tumor specimens in the Department of Pathology collected between 1972 and the beginning of 1987 that had associated complete clinical information. Most of these patients have been included in previous studies evaluating antineoplastic therapies and the prognostic importance of clinical and biochemical correlates of improved patient survival (9). While the patients participating in these clinical trials received a variety of diagnostic, therapeutic, and supportive interventions, the results of these studies did not demonstrate major differences in survival based on the therapeutic regimens used to treat these patients.

Immunohistochemical analysis. Serial 5- $\mu \mathrm{m}$ sections of tumor specimens were analyzed for tyrosine hydroxylase $(\mathrm{TH})^{1}$, chromogranin $\mathrm{A}$

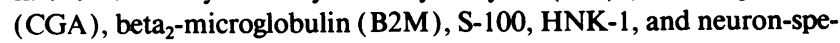

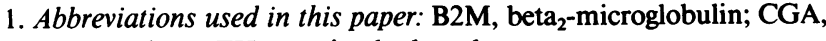
chromogranin $\mathrm{A} ; \mathrm{TH}$, tyrosine hydroxylase. 
cific enolase immunoreactivity using an avidin-biotin complex, horseradish peroxidase-linked detection system (Vectastain $A B C$ peroxidase kit; Vector Labs, Inc., Burlingame, CA) as previously reported (17, 23). To facilitate analysis of chromaffin and ganglionic markers in discrete tumor cell populations, the section stained for HNK-1 immunoreactivity was adjacent to those stained for expression of CGA and TH. In no case were we able to find cells that expressed markers of both lineages. Primary antibodies included a rabbit anti-bovine tyrosine hydroxylase antiserum (Eugene Tech International, Allendale, NJ) used according to the manufacturer's recommendations; a mouse $\mathrm{IgG}_{1}$ anti-human chromogranin A monoclonal antibody (clone LKH210) used at a concentration of $10 \mu \mathrm{g} / \mathrm{ml}$ (Boehringer Mannheim Biochemicals, Indianapolis, IN); a rabbit anti-human B2M antisera (Dako Laboratories, Carpinteria, CA) used at a dilution of 1:100; a rabbit anti-bovine brain S-100 antisera used at a dilution of 1:1,500 (Accurate Chem. \& Sci. Corp., Westbury, NY); HNK-1, a mouse IgM monoclonal antibody used at a dilution of $10 \mu \mathrm{g} / \mathrm{ml}$ ( Becton Dickinson Immunocytometry Systems, Mountain View, CA); and a rabbit anti-human neuron specific enolase antisera used at a dilution of 1:500 (Accurate Chem. \& Sci. Corp.). Control antibodies included isotype matched mouse antimouse $\mathrm{H} 2$ antigen-specific monoclonal antibodies (provided by Dr. David Sachs, National Institutes of Health, Bethesda, MD) and purified rabbit immunoglobulin (Accurate Chem. \& Sci. Corp.). Diaminobenzidine $(0.05 \%)$ (Sigma Chemical Co., St. Louis, MO) was used as the chromogen, and the specimens were counterstained with hematoxylin and eosin using standard techniques. Specimens were examined for marker expression and given final assignments before obtaining survival data. A tumor specimen was scored as positive for a marker if $>5 \%$ of tumor cells in multiple high power fields were immunoreactive. Tumor specimens were categorized as "null" if they lacked any lineage-related marker expression. Nevertheless, all tumor samples, including those in the null category, expressed the nervous system marker, neuron-specific enolase.

Statistical methods. Correlations between the expression of lineage-related markers and prognostic indicators of neuroblastoma patient survival were determined separately; lineage assignment was evaluated for each prognostic variable using the chi-square test. Because 16 comparisons were involved in this analysis, individual $P_{2}$ values $<0.0031$ were considered significant (using a nominal 0.05 level for significance and a rigorous Bonferroni approach) (24). However, $P$ values $<0.01$ are highly suggestive of an association.

Survival durations were computed from the date the patient was diagnosed with neuroblastoma until the date of death or last follow-up. Probabilities of survival as a function of time were determined by the Kaplan-Meier method (25). The Mantel-Haenszel procedure (26) was used to evaluate the usefulness of each prognostic factor (i.e., age, stage, markers of differentiation, Shimada grade, and serum ferritin levels) in predicting patient survival. This procedure was also used to determine the significance of the difference between a pair of Kaplan-Meier curves. The Cox proportional hazards modeling technique (27) was used to identify which factors, when simultaneously evaluated, may have statistical significance in predicting patient survival. The relative risk of dying during any short interval of time for each variable included in a Cox model was determined as $\exp \left(b_{i}\right)$, where $b_{i}$ is the resulting model parameter estimate and $\exp (\mathrm{a})=2.71828^{\mathrm{a}}(27)$.

\section{Results}

We have previously presented a model of human adrenal medullary histogenesis that incorporates the chromaffin, ganglionic, and sustentacular lineages known to constitute the parenchymal cells of the adult adrenal medulla (Fig. 1) (17, 23). CGA, TH, and B2M are markers of the chromaffin lineage; $\mathrm{S}-100$ is a marker of sustentacular cells; and HNK-1 is a marker of fetal adrenal medullary ganglion cells. Within the chromaffin lineage, TH and CGA are expressed early during the first trimester of development, whereas B2M is not expressed until

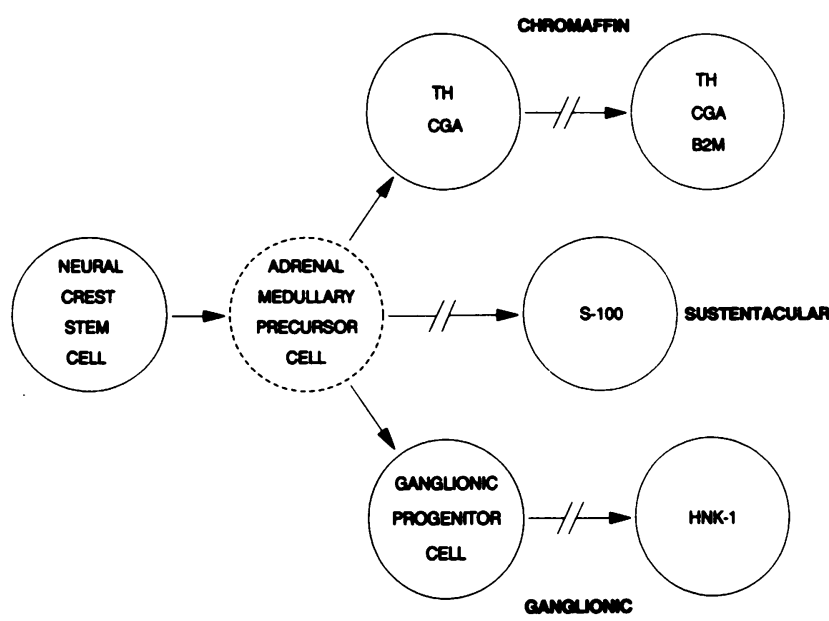

Figure 1. Model of human fetal adrenal medullary histogenesis (23). The adrenal medulla is derived from primitive, multipotent neural crest stem cells. Descendants of these cells that invade the fetal adrenal cortex and give rise to the adrenal medulla are designated as adrenal medullary precursor cells. Since the precise developmental potential of such precursor cells has not been defined, it is represented using a dashed line. Stages in maturation of the chromaffin, sustentacular, and ganglionic lineages are indicated $(17,23)$. Both sustentacular and ganglion cells are thought to arise from nonchromaffin adrenal neuroblasts (23). Sustentacular cells are S-100 positive, spindle-shaped cells that interdigitate among the chromaffin cells. An early stage in ganglionic maturation is represented by the HNK-1 negative, ganglionic progenitor cell. Later during development fetal ganglion cells become HNK-1 positive. Most fetal adrenal ganglion cells undergo developmental cell death, although some survive to become adult adrenal medullary ganglion cells (23). The dashed arrows indicate that intermediary cell types exist, as previously described $(17,23)$.

just before birth. HNK-1 marks a fetal ganglionic lineage that appears to undergo developmental cell death at $\sim 24 \mathrm{wk}$ of gestation (23). This antibody also marks a small percentage of chromaffin adrenal medullary cells beginning $\sim 4-8$ mo after birth, but does not react with most chromaffin cells until after 5 yr of age (23). In neuroblastoma tumor specimens, HNK-1 immunoreactivity is almost uniformly associated with ganglionic differentiation, based on the lack of chromaffin marker expression in these cell types, as well as the localization of HNK-1 staining to tumor-derived neurophil (our unpublished data).

To determine whether neuroblastoma tumor cells correspond to fetal adrenal medullary progenitor cells that have matured along chromaffin, ganglionic, or sustentacular lineages, we examined the expression of adrenal medullary lineage-related markers in 81 tumor samples obtained from surgical specimens of untreated neuroblastoma patients at the Children's Hospital of Philadelphia between 1972 and 1987. The clinical characteristics of this patient population closely reflect those previously reported for patients with neuroblastoma (Tables I and III $)(6,9)$. The proportion of patients with advanced stage tumors in this cadre is decreased, since the diagnosis of stage IV neruoblastoma can often be made by bone marrow examination alone, and primary tumor specimens were therefore not available for all such patients.

We observed in these specimens that the expression of developmentally regulated markers associated with either the chro- 
maffin or sustentacular lineages were unrelated to most previously described prognostic indicators of patient survival, including the age of the patient at diagnosis, serum ferritin level, and histopathologic classification of the tumor (Table I). In contrast, evidence of ganglionic differentiation or absence of all marker expression, designated as a null category, were associated with multiple prognostic indicators. Of particular interest is the association between stage IVS disease and the ganglionic lineage $\left(P_{2}=0.0055\right)$ and the correlation between the null category and an unfavorable Shimada grading $\left(P_{2}=0.0018\right)$. This later result was anticipated, since all tumors in the null group were histologically undifferentiated and, therefore, would likely score in the unfavorable category of the Shimada classification.

While all tumors in the null category were histologically undifferentiated, not all undifferentiated tumors were null; some expressed markers of neural crest differentiation. Presented in Fig. 2 are tumor specimens from two undifferentiated neuroblastomas evaluated for TH expression $(A$ and $C$ ) and HNK-1 immunoreactivity ( $B$ and $D$ ). These small, round, blue cell tumors are histologically indistinguishable from each other and from other null category tumors. The tumor in Fig. 2, $C$ and $D$ has differentiated along a chromaffin pathway, as indicated by brown immunoperoxidase staining of tumor cells for TH. In contrast, the tumor in Fig. 2, $A$ and $B$ has differentiated along a ganglionic lineage, as indicated by HNK-1 immunoreactivity without TH expression. These findings demonstrate that histologically indistinguishable neuroblastoma tumors can be recognized to have evidence of differentiation along different lineages and indicate that tumor cells may differentiate along distinct lineages in the absence of morphologic evidence of tumor cell maturation.

Table I. Correlation between the Expression of Lineage-related Markers and Prognostic Indicators of Neuroblastoma Patient Survival

\begin{tabular}{lccccc}
\hline \multicolumn{5}{c}{ Number of tumors expressing lineage-related markers } \\
\hline Variable* & $\begin{array}{c}\text { Tumors } \\
\text { analyzed }\end{array}$ & Chromaffin & Sustentacular & Ganglionic & Null \\
\hline Age & & & & & \\
$<1$ yr & 36 & 24 & 9 & 25 & 3 \\
$\geq 1$ yr & 45 & 28 & 22 & 21 & 12 \\
Stage & & & & & \\
I, II & 32 & 22 & 15 & $20^{\ddagger 5}$ & 5 \\
III & 16 & 8 & 6 & 9 & 5 \\
IV & 24 & 16 & 9 & 8 & 5 \\
IVS & 9 & 6 & 1 & 9 & 0 \\
Ferritin & & & & & \\
Low & 61 & 42 & 25 & 39 & $7^{\| \prime}$ \\
High & 19 & 9 & 6 & 6 & 8 \\
Shimada & & & & & \\
Favorable & 43 & 31 & 22 & $31^{\prime \prime}$ & $3^{\prime \prime}$ \\
$\quad$ Unfavorable & 34 & 17 & 8 & 13 & 12 \\
\hline
\end{tabular}

* The stage reported is the Evans staging system (28). Patients with serum ferritin levels $>150 \mathrm{ng} / \mathrm{ml}$ were considered elevated (9). Tumors were classified according to the Shimada grading system as previously reported (11). ${ }^{\ddagger}$ Groupings by stage are as follows: I + II, III $+\mathrm{IV}, \operatorname{IVS}\left(P_{2}=0.005\right) ; \mathrm{I}+\mathrm{II}+\mathrm{III}+\mathrm{IV}, \operatorname{IVS}\left(P_{2}=0.0055\right) .{ }^{\S} 0.0031$ $\leq P_{2}<0.01$. " $P_{2}<0.0031$ (significant, see text).
Although the tumor specimens presented in Fig. 2 are composed of cells that have differentiated along a single lineage, other tumor specimens were composed of groups of cells that expressed markers from different adrenal medullary lineages. Populations of chromaffin and nonchromaffin tumor cells tended to be localized to different areas of the tumor specimen (data not shown). Of the 66 tumors that expressed lineage-related markers, $30 \%$ expressed markers from a single lineage, whereas $70 \%$ expressed markers from multiple lineages (data not shown). Since neuroblastoma is thought to be a clonal tumor (29), this finding suggests that some neuroblastoma tumor cells retain the plasticity to differentiate along multiple developmental pathways.

Most neuroblastoma cell lines appear to correspond to adrenal medullary chromaffin progenitor cells arrested at one of several different stages of maturation (17). We therefore evaluated the expression of several developmentally regulated chromaffin markers in neuroblastoma tumor specimens. Presented in Table II are the numbers of tumor specimens that expressed either markers of early chromaffin adrenal medullary maturation, TH and / or CGA, or a marker of mature embryonal chromaffin adrenal medullary cells, B2M. All B2M positive tumor specimens also expressed the early chromaffin markers. Significantly, there were no examples of tumor specimens that expressed B2M without coexpression of early chromaffin markers $\left(P_{2}<10^{-5}\right)$, the single combination that would have been inconsistent with the pattern of markers expressed during normal development. This finding indicates that chromaffin markers are not expressed in a random fashion. Rather, the observed pattern of gene expression closely mimics that found during embryonal development of chromaffin adrenal medullary cells.

These data indicate that neuroblastoma tumor cells may correspond to the arrested differentiation of adrenal medullary precursors at different points along maturational pathways. We reasoned that the maturational stage or the lineage to which the tumor corresponds may correlate with distinct tumor cell properties, including various features of the tumor that are of clinical significance. We evaluated this possibility by examining the prognostic value of lineage-related marker expression, as well as other previously described prognostic indicators, including age at diagnosis, stage, serum ferritin level, and the Shimada classification. The N-myc copy number and DNA ploidy analysis were not available for most of these patients. As summarized in Table III, we found that evidence of differentiation along a fetal ganglionic lineage, as marked by HNK-1 immunoreactivity, was a significant prognostic variable in this univariate analysis, comparable to these other prognostic indicators. The expression of other developmentally regulated markers was not of prognostic significance.

To evaluate if HNK-1 was an independent prognostic indicator of patient survival, we performed a Cox proportional hazards analysis including patient age at diagnosis, stage, serum ferritin level, and lineage-related marker expression in the models. As the Shimada classification incorporates the age of the patient at diagnosis in its formulation, it could not be considered independent of age, and therefore was not included in this analysis. The most predictive model we could identify (model chi square value of 44.25 with three degrees of freedom) included only three variables: HNK-1 immunoreactivity, with a relative risk of 6.42 for those patients having HNK-1 negative tumors $\left(P_{2}=0.0001\right)$; age at diagnosis, with a relative 


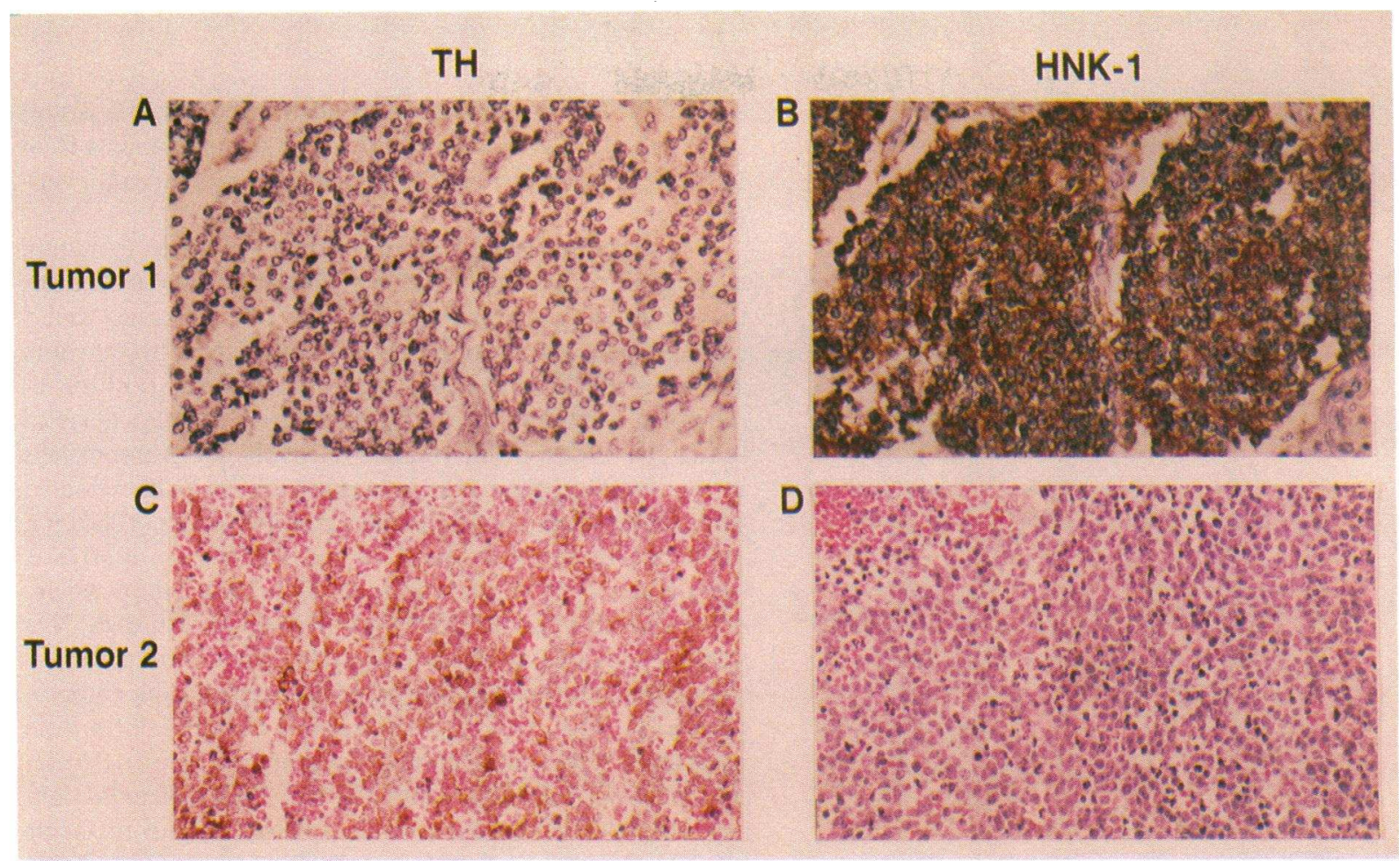

Figure 2. Histologically indistinguishable neuroblastoma tumors have different patterns of lineage-related gene expression. Two neuroblastoma tumor specimens with undifferentiated histopathologic features (tumors 1 and 2 ) were evaluated by immunoperoxidase staining for TH expression $(A$ and $C)$ and HNK-1 $(B$ and $D)$ immunoreactivity. $\times 190$.

risk of 5.05 for those patients $>12$ mo vs those $<12$ mo of age $\left(P_{2}=0.0042\right)$; and serum ferritin which had less significance, with a relative risk of 2.20 for those patients having high levels $\left(P_{2}=0.0513\right)$. The independent prognostic significance of age at diagnosis and HNK-1 immunoreactivity is consistent with the lack of correspondence between age and evidence of differentiation along the ganglionic lineage, as presented in Table $\mathbf{I}$.

The Kaplan-Meier survival curves in Fig. 3, $A$ and $B$, illustrate the independent prognostic significance of both age at diagnosis and HNK-1 immunoreactivity. The survival of patients $>1 \mathrm{yr}$ of age at diagnosis, a widely recognized indicator of poor prognosis, is presented in $(A)$ according to the presence or absence of HNK-1 immunoreactivity in their tumor specimens. Patients with HNK-1 positive tumors had a better prognosis than patients with HNK-1 negative tumors $\left(P_{2}<10^{6}\right)$. The 5-yr survival of patients with HNK-1 positive tumors was $81 \%$, whereas the 5-yr survival of patients with HNK-1 negative tumors was $12 \%$. Similarly, the survival of patients with HNK-1 negative tumors, a bad prognostic category, is presented in Fig. $3 B$ according to the age of the patient at diagnosis. Patients $<1$ yr of age had a better prognosis than older patients $\left(P_{2}=0.0008\right)$. The 5 -yr survival of patients $<1 \mathrm{yr}$ of age was $82 \%$, whereas the 5 -yr survival of patients $>1$ yr of age was $12 \%$.

In contrast to HNK-1 and age of the patient at diagnosis, stage was not an independent predictor of survival in our multivariate Cox proportional hazard analysis. This finding is illustrated by the Kaplan-Meier survival curves in (Fig. 3, $C$ and $D)$. As shown in $(C)$, advanced stage patients having HNK-1 positive tumors have a better survival than patients having HNK-1 negative tumors $\left(P_{2}=0.0008\right)$. However, among patients having HNK-1 negative tumors $(D)$, stage was not able to identify patient subsets having a good prognosis with the same ability $\left(P_{2}=0.026\right)$.

\section{Discussion}

Although numerous lines of investigation indicate that malignant cells from aggressive solid tumors often display poorly differentiated features (30), there is little evidence suggesting a correspondence between solid tumor cells and the cell types found during histogenesis of tissues in which such tumors arise. The availability of multiple chromaffin-related developmental markers made it possible to critically evaluate the hypothesis that neuroblastoma tumor cells correspond to the arrested differentiation of chromaffin adrenal medullary progenitor cells. As reported in Table II, we found no example of chromaffin-related marker expression in neuroblastoma tumor specimens that deviated from the normal developmental pattern of gene expression. These findings indicate that chromaffin gene expression in neuroblastoma is not a stochastic process. Rather, these data strongly argue that some of the regulatory mechanisms that control chromaffin maturation in normal adrenal

Table II. Neuroblastoma Tumor Cells Correspond to the Arrested Differentiation of Chromaffin Adrenal Medullary Progenitor Cells

\begin{tabular}{|c|c|c|}
\hline \multicolumn{2}{|c|}{ Chromaffin markers* } & \multirow{2}{*}{$\begin{array}{l}\text { Tumors with designated pattern } \\
\text { of marker expression }\end{array}$} \\
\hline Early (TH/CGA) & Late (B2M) & \\
\hline- & - & 29 \\
\hline- & + & 0 \\
\hline+ & - & 26 \\
\hline+ & + & 26 \\
\hline
\end{tabular}

* The markers evaluated included TH, CGA, and B2M. +, detectable immunoreactivity; -, no immunoreactivity was detected. ${ }^{\ddagger} P_{2}$ $<10^{-5}$ by the chi-square test. 
Table III. Univariate Analysis of Lineage-related Marker Expression and Standard Prognostic Indicators in Predicting Survival of Patients with Neuroblastoma

\begin{tabular}{|c|c|c|c|}
\hline Variable & Number & $\begin{array}{c}5 \text {-yr survival } \\
(\%)^{*}\end{array}$ & $P_{2}$ \\
\hline \multicolumn{4}{|l|}{ Age } \\
\hline$<1$ Year & 36 & 89 & \multirow[t]{2}{*}{$<0.0001$} \\
\hline$\geq 1$ Year & 45 & 44 & \\
\hline \multicolumn{4}{|l|}{ Stage } \\
\hline I, II & 32 & 81 & \multirow{4}{*}{$0.0026^{\ddagger}$} \\
\hline III & 16 & 56 & \\
\hline IV & 24 & 38 & \\
\hline IVS & 9 & $89^{\S}$ & \\
\hline \multicolumn{4}{|l|}{ Ferritin } \\
\hline Low & 61 & 77 & \multirow[t]{2}{*}{$<0.0001$} \\
\hline High & 19 & 26 & \\
\hline \multicolumn{4}{|l|}{ Shimada } \\
\hline Favorable & 43 & 91 & \multirow[t]{2}{*}{$<0.0001$} \\
\hline Unfavorable & 34 & 38 & \\
\hline \multicolumn{4}{|l|}{$\mathrm{TH}$} \\
\hline Positive & 47 & 68 & \multirow[t]{2}{*}{0.48} \\
\hline Negative & 34 & 59 & \\
\hline \multicolumn{4}{|l|}{ CGA } \\
\hline Positive & 18 & 66 & \multirow[t]{2}{*}{0.93} \\
\hline Negative & 63 & 63 & \\
\hline \multicolumn{4}{|l|}{ B2M } \\
\hline Positive & 26 & 57 & \multirow[t]{2}{*}{0.94} \\
\hline Negative & 55 & 64 & \\
\hline \multicolumn{4}{|l|}{$S-100$} \\
\hline Positive & 31 & 70 & \multirow[t]{2}{*}{0.48} \\
\hline Negative & 50 & 60 & \\
\hline \multicolumn{4}{|l|}{ HNK-1 } \\
\hline Positive & 46 & 83 & \multirow[t]{2}{*}{$<0.0001$} \\
\hline Negative & 35 & 34 & \\
\hline
\end{tabular}

* Median follow up of survivors is $86 \mathrm{mo}$. ${ }^{\ddagger}$ Stages I + II compared to stages III and IV. ${ }^{\S}$ The sample size for this group is small; $95 \%$ confidence interval is $57-98 \%$.

embryonal neuroblasts are operative in neuroblastoma tumor cells.

The lack of multiple sustentacular and ganglionic markers did not allow us to determine if neuroblastoma tumor cells also correspond to the arrested maturation of adrenal medullary progenitor cells that have differentiated along these pathways. Nevertheless, S-100 and HNK-1 immunoreactivity provides an assay to determine if a tumor cell has matured along nonchromaffin pathways. As presented in Table I, many tumor specimens expressed nonchromaffin markers, suggesting that these developmental pathways of neural crest origin, as well as the chromaffin lineage, are important in the biology of this tumor.

This developmental analysis of neuroblastoma has identified a differentiation pathway associated with tumors that are responsive to antineoplastic therapies and/or lack aggressive potential. Patients with tumors that had immunohistochemical evidence of ganglionic differentiation had significantly improved survival compared to patients having HNK-1 negative tumors (Table III). As illustrated in Fig. 3, both age at diagno- sis and HNK-1 immunoreactivity can independently distinguish subsets of good prognosis neuroblastoma patients from larger groups of high risk patients defined by use of either variable alone.

Many neuroblastoma tumors retain the plasticity to mature along multiple adrenal medullary lineages. Of the 66 tumors that expressed lineage-related markers, $70 \%$ contained different populations of cells expressing markers from two or more adrenal medullary lineages. Patients with HNK-1 positive tumors had a good prognosis irrespective of the percentage of tumor cells expressing this marker or whether other tumor cells in the specimen were marker negative or had differentiated along chromaffin or sustentacular pathways (data not shown). These data suggest that the biological significance of HNK-1 immunoreactivity in predicting patient survival is the plasticity of tumor cells to mature along a fetal ganglionic lineage. This tumor plasticity may be mediated through intrinsic pathways characteristic of neural crest progenitor cells during embryogenesis $(31,32)$, and may result from the presence of extrinsic modulators of neural crest cell differentiation $(33,34)$. Several agents that can modulate adrenal medullary differentiation, including glucocorticoids (35-37), fibroblast growth factor $(38,39)$, and nerve growth factor (35-37) have been identified in model systems of neural crest maturation. In addition, the presence of local growth factors normally produced by the adrenal cortex, such as IGF-II, may also influence the proliferation and differentiation of neuroblastoma tumor cells (40).

The association between HNK-1 immunoreactivity and improved patient survival suggests that therapeutic strategies to differentiate neuroblastoma tumors along a ganglionic lineage may be successful in modulating the therapeutic responsiveness of these tumors. In vitro studies using neuroblastoma cell lines indicate that some biological response modifiers, such as retinoic acid and dexamethasone, can modulate chromaffinrelated marker expression (41-42) (data not shown). Related studies may identify subsets of neuroblastoma patients whose tumor cells retain the plasticity to mature along a ganglionic pathway, suggesting a novel approach for the use of biological response modifiers to modulate tumor cell biology and render currently incurable patients sensitive to available antineoplastic therapies.

This developmental analysis of neuroblastoma also suggests a mechanism for the spontaneous regression of stage IVS tumors. In previous studies, we have reported that the number of ganglion progenitor cells in fetal adrenal glands dramatically falls after $24 \mathrm{wk}$ of gestation (23). Since many fetal ganglion cells have evidence of nuclear fragmentation at this developmental stage (data not shown), we believe that most fetal adrenal ganglion cells undergo developmental cell death. As presented in Table I, our analysis revealed that all nine stage IVS tumor specimens were HNK-1 positive compared to 37 of 72 non-stage IVS tumors, an association with near statistical significance in view of the numerous comparisons being made in this retrospective analysis $\left(P_{2}=0.0055\right)$. These data raise the possibility that the spontaneous remission of stage IVS disease may be related to mechanisms underlying the programmed cellular death of fetal adrenal medullary ganglion cells. The potential to induce tumor cells to undergo cell death might well be pursued in neuroblastoma.

The composition of our patient population by age and stage is essentially identical to other reports $(6,43)$, except that stage IV patients account for $30 \%$ of our population compared to 
A

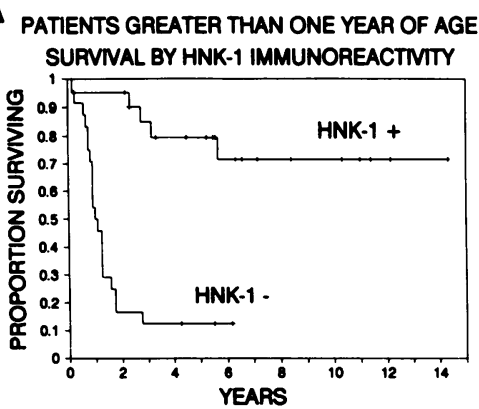

B

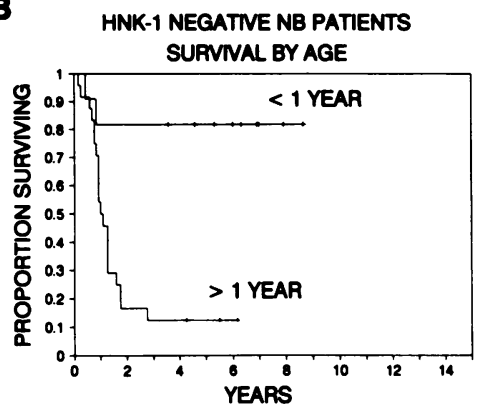

C

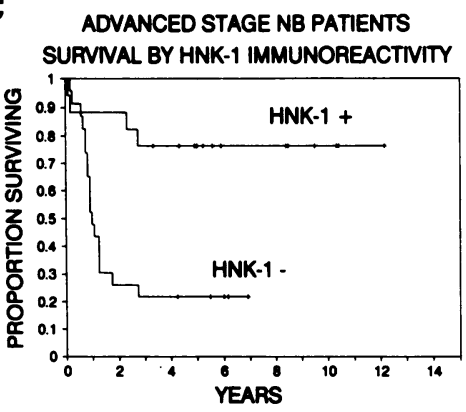

D

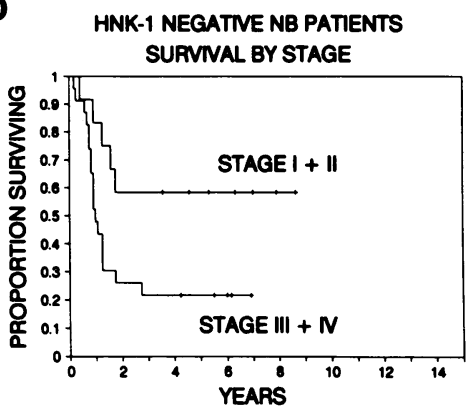

Figure 3. Survival of neuroblastoma patients by age at diagnosis, stage, and HNK-1 immunoreactivity. $(A)$ Kaplan-Meier survival curves of patients $>1$ year of age $(n=45)$ by presence or absence of HNK-1 immunoreactivity $\left(P_{2}<10^{-6}\right)$. $(B)$ Kaplan-Meier survival curves of patients having HNK-1 negative tumors $(n=35)$ by age at diagnosis $\left(P_{2}=0.0008\right)$. (C) shows the survival of advanced stage neuroblastoma patients (stages III and IV, $n=40$ ) by presence or absence of HNK-1 immunoreactivity $\left(P_{2}=0.0008\right)$. $(D)$ Kaplan-Meier survival curves of patients having HNK-1 negative tumors $(n=35)$ by stage $\left(P_{2}\right.$ $=0.026)$. $\sim 40 \%$ in other studies. This finding likely reflects our decision to include only patients having biopsies of their primary tumors, since we currently do not know whether primary tumors and metastatic sites will have concordant patterns of developmental marker expression. Therefore, those stage IV patients diagnosed solely on the basis of a positive bone marrow biopsy were excluded from our analysis. Additionally, our stage IV patients had a better 5 -yr survival rate (38\%, Table III) than previously tabulated by Breslow and Bernstein $(\sim 5-20 \%)(6$, 43). While subset analysis of our data excluding stage IV patients did not change the correlation between HNK-1 immunoreactivity and improved patient survival (data not shown), it will be important to test the prognostic significance of HNK-1 immunoreactivity in a complete cohort of prospectively accrued neuroblastoma patients.

In summary, these studies demonstrate that neuroblastoma tumors consist of cells that correspond to embryonal adrenal medullary progenitors arrested at recognizable stages in their maturation; that evidence of maturation along a fetal ganglionic lineage appears to have major importance in predicting patient survival; and that biologically distinct subgroups of the same tumor type that have differing responses to antineoplastic therapy and/or vary in their malignant potential can be recognized by an evaluation of lineage-related gene expression. It is possible that other pediatric solid tumors may also correspond to the arrested differentiation of tissue progenitor cells, and recent reports suggest that rhabdomyosarcoma and Wilm's tumor may fit this paradigm $(44,45)$. Although it is currently unknown whether common adult tumors, such as colon, lung, and bladder cancers, correspond to the arrested maturation of epithelial cells at different stages of cell renewal and differentiation, it is conceivable that the complex biology of these tumor types may be related to distinct biological properties of epithelial progenitor cells at different stages of tissue maturation.

\section{Acknowledgments}

We gratefully acknowledge the generous support of this research by the Preuss Foundation.

\section{References}

1. Le Douarin, N. M. 1984. The neural crest. In The Developmental and Cell Biology Series. P. W. Barlow, P. B. Green, C. C. Wylie, editors. Cambridge University Press, Cambridge, England. 134-169.

2. Triche, T. J., and F. B. Askin. 1983. Neuroblastoma and the differential diagnosis of small-, round-, blue-cell tumors. Hum. Pathol. 14:569-595.

3. Fox, F., J. Davidson, and L. B. Thomas. 1959. Maturation of sympathicoblastoma into ganglioneuroma. Cancer. 12:108-116.

4. Tsokos, M., S. Scarpa, R. Ross, and T. J. Triche. 1987. Differentiation of human neuroblastoma recapitulates neural crest development. Study of morphology, neurotransmitter enzymes, and extracellular matrix proteins. Am. J. Pathol. 128:484-496.

5. Reynolds, C. P., and J. Maples. 1986. Modulation of cell surface antigens accompanies morphological differentiation of human neuroblastoma cell lines. Prog. Clin. Biol. Res. 271:13-37.

6. Breslow, N., and B. McCann. 1971. Statistical estimation of prognosis for children with neuroblastoma. Cancer Res. 31:2098-2103.

7. Philip, T., J. L. Bernard, J. M. Zucker, R. Pinkerton, P. Lutz, P. Bordigoni, E. Plouvier, A. Robert, R. Carton, N. Philippe et al. 1987. High dose chemoradiotherapy with bone marrow transplantation as consolidation treatment in neuroblastoma: an unselected group of stage IV patients over 1 year of age. J. Clin. Oncol. 5:266-271.

8. Pritchard, J., E. Kiely, D. W. Rogers, L. Spitz, E. A. Shafford, R. Brereton, C. Muller, and V. M. Wright. 1987. Long-term survival after advanced neuroblastoma. N. Engl. J. Med. 317:1026-1027.

9. Evans, A. E., G. J. D'Angio, K. Propert, J. Anderson, and H. W. Hann. 1987. Prognostic factors in neuroblastoma. Cancer. 59:1853-1859.

10. Seeger, R. C., G. M. Brodeur, H. Sather, A. Dalton, S. E. Siegel, K. Y. Wong, and D. Hammond. 1985. Association of multiple copies of the N-myc oncogene with rapid progression of neuroblastomas. N. Engl. J. Med. 313:11111116.

11. Shimada, H., C. Aoyama, T. Chiba, and W. A. Newton. 1985. Prognostic subgroups for undifferentiated neuroblastoma: immunohistochemical study with anti-S-100 protein antibody. Hum. Pathol. 16:471-476.

12. Hayashi, Y., R. Ohi, M. Sone, K. Takahashi, T. Mouri, T. Watanabe, S. Yaoita, and M. Nakamura. 1991. Significance of plasma neuropeptide Y (NPY) in diagnosis and prognosis of neuroblastoma. Prog. Clin. Biol. Res. 366:359-365.

13. Kogner, P., E. Theodorsson, and O. Bjork. 1991. Plasma neuropeptide $Y$ (NPY): a novel marker of neuroblastoma. Prog. Clin. Biol. Res. 366:367-373.

14. Beckwith, J. B., and E. V. Perrin. 1963. In situ neuroblastomas: a contribution to the natural history of neural crest tumors. Am. J. Pathol. 43:1089-1104.

15. Ikeda, Y., J. Lister, J. M. Bouton, and M. Buyukpamukcu. 1981. Congenital neuroblastoma, neuroblastoma in situ, and the normal fetal development of the adrenal. J. Pediatr. Surg. 16:636-644.

16. Sidell, N. 1982. Retinoic acid-induced growth inhibition and morphologic differentiation of human neuroblastoma cells in vitro. J. Natl. Cancer Inst. 68:589-596.

17. Cooper, M. J., G. M. Hutchins, P. S. Cohen, L. J. Helman, R. J. Mennie, and M. A. Israel. 1990. Human neuroblastoma tumor cell lines correspond to the 
arrested differentiation of chromaffin adrenal medullary neuroblasts. Cell Growth \& Differ. 1:149-159.

18. Ciccarone, V., B. A. Spengler, M. B. Meyers, J. L. Biedler, and R. A. Ross. 1989. Phenotypic diversification in human neuroblastoma cells: expression of distinct neural crest lineages. Cancer Res. 49:219-225.

19. D'Angio, G. J., A. E. Evans, and C. E. Koop. 1971. Special pattern of widespread neuroblastoma with a favourable prognosis. Lancet. i:1046-1049.

20. Evans, A. E., J. Chatten, G. J. D'Angio, J. M. Gerson, J. Robinson, and L. Schnaufer. 1980. A review of 17 IV-S neuroblastoma patients at the Children's Hospital of Philadelphia. Cancer. 45:833-839.

21. Evans, A. E., J. Gerson, and L. Schnaufer. 1976. Spontaneous regression of neuroblastoma. Natl. Cancer Inst. Monogr. 44:49-54.

22. Cooper, M. J., G. M. Hutchins, R. J. Mennie, and M. A. Israel. 1990 Beta-2-microglobulin expression in embryonal neuroblastoma reflects its developmental regulation. Cancer Res. 50:3694-3700.

23. Cooper, M. J., G. M. Hutchins, and M. A. Israel. 1990. Histogenesis of the human adrenal medulla: an evaluation of the ontogeny of chromaffin and nonchromaffin lineages. Am. J. Pathol. 137:605-615.

24. Matthews, D. E., and V. T. Farewell. 1985. Using and Understanding Medical Statistics. S. Karger, A. G., Basel. 158-172.

25. Kaplan, E., and P. Meier. 1958. Non-parametric estimation from incomplete observations. J. Am. Stat. Assoc. 53:457-81.

26. Mantel, N. 1966. Evaluation of survival data and two new rank order statistics arising in its consideration. Cancer Chemother. Rep. 50:163-70.

27. Cox, D. 1972. Regression models and lifetables. J. R. Stat. Soc. (B) 34:187-202.

28. Evans, A. E., G. J. D'Angio, and J. Randolph. 1971. A proposed staging for children with neuroblastoma. Cancer. 27:374-378.

29. Gilbert, F., G. Balaban, P. Moorhead, D. Bianchi, and H. R. Schlesinger. 1982. Abnormalities of chromosome $1 \mathrm{p}$ in human neuroblastoma tumors and cell lines. Cancer Genet. Cytogenet. 7:33-42.

30. Cotran, R. S., V. Kumar, and S. L. Robbins. 1982. Neoplasia. In Pathologic Basis of Disease. R. S. Cotran, V. Kumar, and S. L. Robbins, editors. W. B. Saunders Company, Philadelphia. 239-305.

31. Bronner-Fraser, M., and S. E. Fraser. 1988. Cell lineage analysis reveals multipotency of some avian neural crest cells. Nature (Lond.). 335:161-164.

32. Baroffio, A., E. Dupin, and N. M. Le Douarin. 1988. Clone-forming ability and differentiation potential of migratory neural crest cells. Proc. Natl. Acad. Sci. USA. 85:5325-5329.

33. Anderson, D. J. 1989. The neural crest cell lineage problem: neuropoiesis? Neuron. 3:1-12.
34. Patterson, P. H. 1990. Control of cell fate in a vertebrate neurogenic lineage. Cell. 62:1035-1038.

35. Doupe, A. J., S. C. Landis, and P. H. Patterson. 1985. Environmental influences in the development of neural crest derivatives: glucocorticoids, growth factors, and chromaffin cell plasticity. J. Neurosci. 5:2119-2142.

36. Doupe, A. J., P. H. Patterson, and S. C. Landis. 1985. Small intensely fluorescent cells in culture: role of glucocorticoids and growth factors in their development and interconversions with other neural crest derivatives. J. Neurosci. 5:2143-2160.

37. Anderson, D. J., and R. Axel. 1986. A bipotential neuroendocrine precursor whose choice of cell fate is determined by NGF and glucocorticoids. Cell. 47:1079-1090.

38. Stemple, D. L., N. K. Manhanthappa, and D. J. Anderson. 1988. Basic FGF induces neuronal differentiation, cell division, and NGF dependence in chromaffin cells: a sequence of events in sympathetic development. Neuron. $1: 517-525$.

39. Claude, P., I. M. Parada, K. A. Gordon, P. A. D'Amore, and J. A. Wagner 1988. Acidic fibroblast growth factor stimulates adrenal chromaffin cells to proliferate and to extend neurites, but is not a long-term survival factor. Neuron. 1:783-790.

40. El-Badry, O. M., J. A. Romanus, L. J. Helman, M. J. Cooper, M. M. Rechler, and M. A. Israel. 1989. Autonomous growth of a human neuroblastoma cell line is mediated by insulin-like growth factor II. J. Clin. Invest. 84:829-839.

41. Cohen, P. S., M. J. Cooper, L. J. Helman, C. J. Thiele, R. C. Seeger, and M. A. Israel. 1990. Neuropeptide $Y$ expression in neonatal neuroblastoma tumors may mimic its developmental regulation in the human adrenal medulla. Cancer Res. 50:6055-6061.

42. Helman, L. J., C. J. Thiele, W. M. Linehan, B. D. Nelkin, S. B. Baylin, and M. A. Israel. 1987. Molecular markers of neuroendocrine development and evidence of environmental regulation. Proc. Natl. Acad. Sci. USA. 84:2336-2339.

43. Bernstein, M. L., J. M. Leclerc, G. Bunin, L. Brisson, L. Robison, J. Shuster, T. Byrne, D. Gregory, G. Hill, G. Dougherty et al. 1991. A populationbased study of neuroblastoma incidence, survival, and mortality in $\mathrm{N}$. Am.J. Clin. Oncol. 10:323-329.

44. Dias, P., D. M. Parham, D. N. Shapiro, B. L. Webber, and P. J. Houghton 1990. Myogenic regulatory protein (MyoD1) expression in childhood solid tumors: diagnostic utility in rhabdomyosarcoma. Am. J. Pathol. 137:1283-1291.

45. Beckwith, J. B., N. B. Kiviat, and J. F. Bonadio. 1990. Nephrogenic rests, nephroblastomatosis, and the pathogenesis of Wilm's tumor. Pediatr. Pathol. 10:1-36. 